\title{
THE SIGNIFICANCE OF VASCULAR CHANGES IN AN ACCESSORY LUNG PRESENTING AS A DIAPHRAGMATIC CYST
}

BY

\author{
D. HEATH AND G. T. WATTS
}

From the Department of Pathology, the Medical School, University of Birmingham, ard the Department of Thoracic Surgery, Queen Elizabeth Hospital, Birmingham

(RECEIVED FOR PUBLICATION DECEMBER 10, 1956)

The purpose of this paper is to describe the pathology of an accessory lung which presented as a diaphragmatic cyst and to assess the significance of vascular changes that occurred in it.

\section{CASE REPORT}

A woman, aged 58 years, was admitted to hospital with a four-year history of dysphagia and indigestion and heart burn which were unrelieved by alkalis and became progressively worse. She first had the sensation of food sticking in the chest in 1952, and in July, 1955, had complete dysphagia for solids. She sought medical advice in September, 1955, and, although clinical examination was negative, a hiatus hernia was demonstrated by a barium meal (Fig. 1). This radiological examination and a teleradiograph of the chest also demonstrated a shadow considered to be produced by

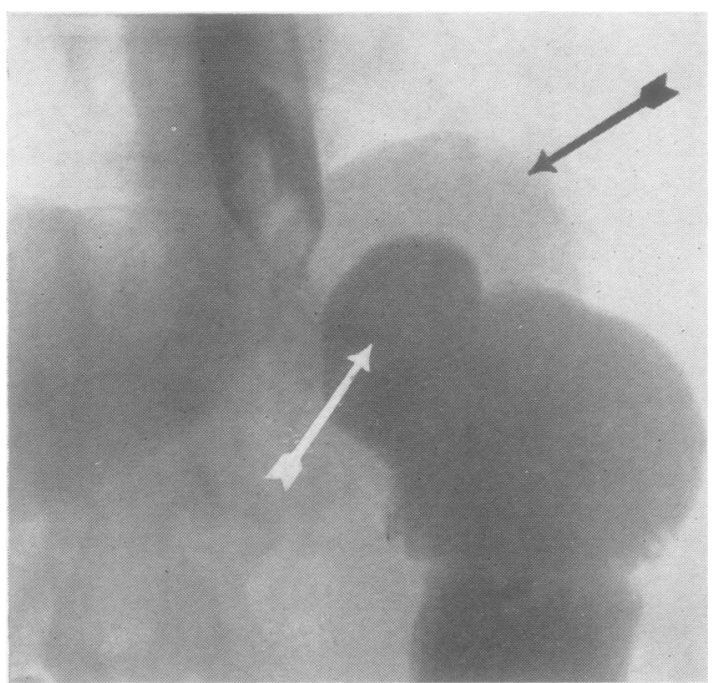

FIG. 1.-Barium meal taken before thoracotomy. The hiatus'hernia is indicated by a white arrow and the outline of the diaphragmatic cyst by a black arrow. a diaphragmatic cyst (Figs. 1 and 2). The systemic blood pressure was $100 / 80 \mathrm{~mm}$. Hg. At first she refused operation, but after persistent dysphagia and vomiting, in June, 1956, she submitted to thoracotomy at which a small hiatus hernia was repaired. At operation a cyst was found embedded in the posterior part of the diaphragm. The cyst was supplied by small branches of the phrenic arteries; there was no single large branch of the aorta supplying the tumour. The cyst was removed.

\section{Pathological Report}

The diaphragmatic cyst, $7 \mathrm{~cm} . \times 2.5 \mathrm{~cm}$., was bunshaped and weighed $94 \mathrm{~g}$. (Fig. 3A). Most of its wall was smooth and so thin as to be translucent, but at one pole it was thick-walled and loculated (Fig. 3B). Both the main cyst cavity and the locules contained a thick, creamy fluid.

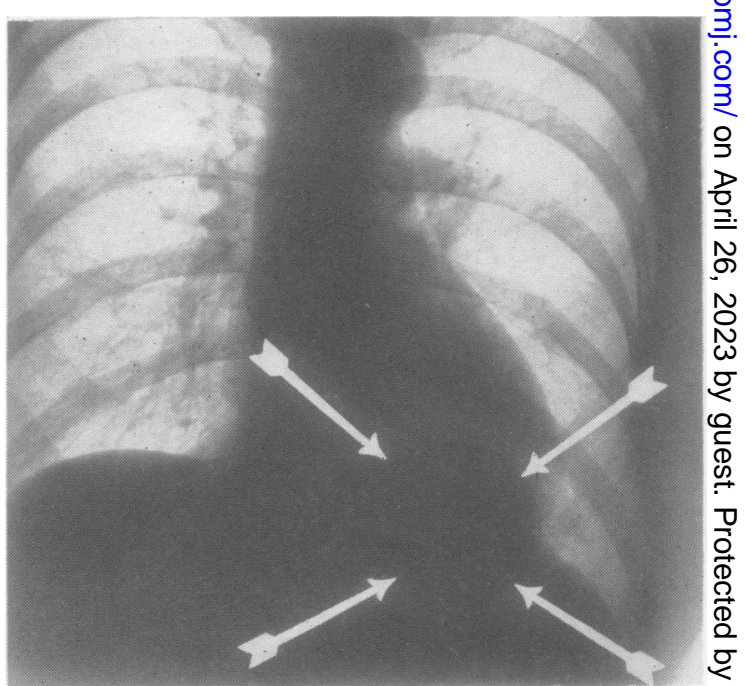

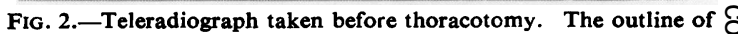
the shadow formed by the diaphragmatic cyst is indicated by white arrows. 


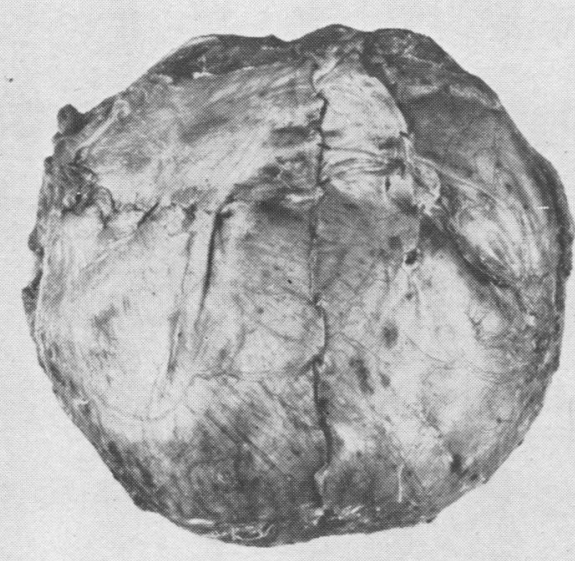

A

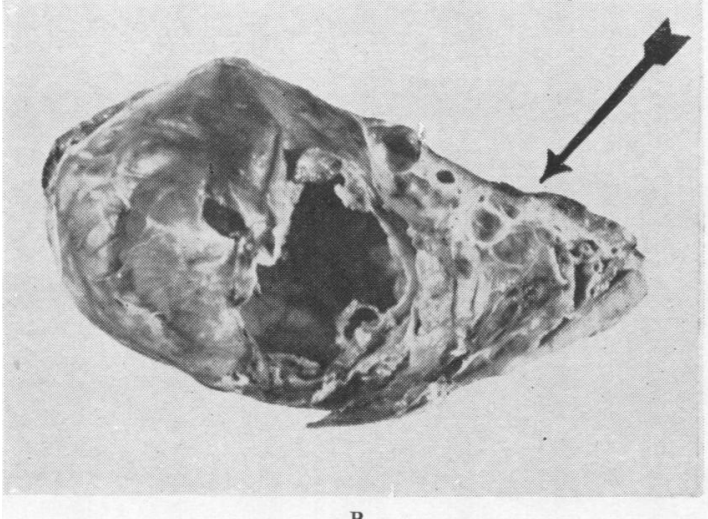

B

FIG. 3.-A. Superior aspect of the cyst (nearly actual size). B. The cyst sectioned to show the smooth, paper-thin wall and the thick, loculated portion at one pole which is indicated by a black arrow (nearly actual size).

Histologically the tumour was composed of cysts which contained inspissated nucus and were lined by pseudostratified ciliated colunınar epithelium; mucussecreting glands and smooth nuuscle were present in the fibrous tissue deep to this and produced an appearance suggestive of groups of bronchi (Fig. 4). A few rudimentary pulmonary alveoli were present together with fragments of calcified material and foci of chronic inflammatory cells.

Blood vessels up to $800 \mu$ in diameter were seen between the dilated bronchi. These had a media containing many concentric elastic fibrils, and internal and external elastic laminae which were fragmented in places (Figs. 5A, 5B). The medial thickness was 10 to $20 \%$ of the external diameter of the vessels. In the intima there was great increase of fibrous tissue containing elastic fibrils; one vessel, $320 \times 250 \mu$, was occluded by this tissue to the extent that its lumen was only $80 \mu$ in diameter. In close association with the abnormal bronchi were arterioles, about $150 \mu$ in diameter, whose medial thickness was $10 \%$ of the external diameter.

Two of the arteries in the cyst showed medial necrosis and one of them, $500 \times 300 \mu$ in diameter (Figs. 5C, 5D), had a medial thickness of $80 \mu$, equal to about $20 \%$ of the average external diameter of the vessel. There was a breach in the external elastic membrane $265 \mu$ in length and the internal elastic membrane over the corresponding length was thin and fragmented in places with portions of elastic fibrils in the necrotic media between the two main laminae. The vascular lumen was occluded by hyaline fibrous tissue. All layers of the artery were infiltrated by leucocytes. The area involved in cellular reaction around this artery, which had fresh haemorrhages in its wall, was approximately $800 \mu$ in diameter (Fig. 5C). The other vessel showing necrosis measured $140 \times 100 \mu$.

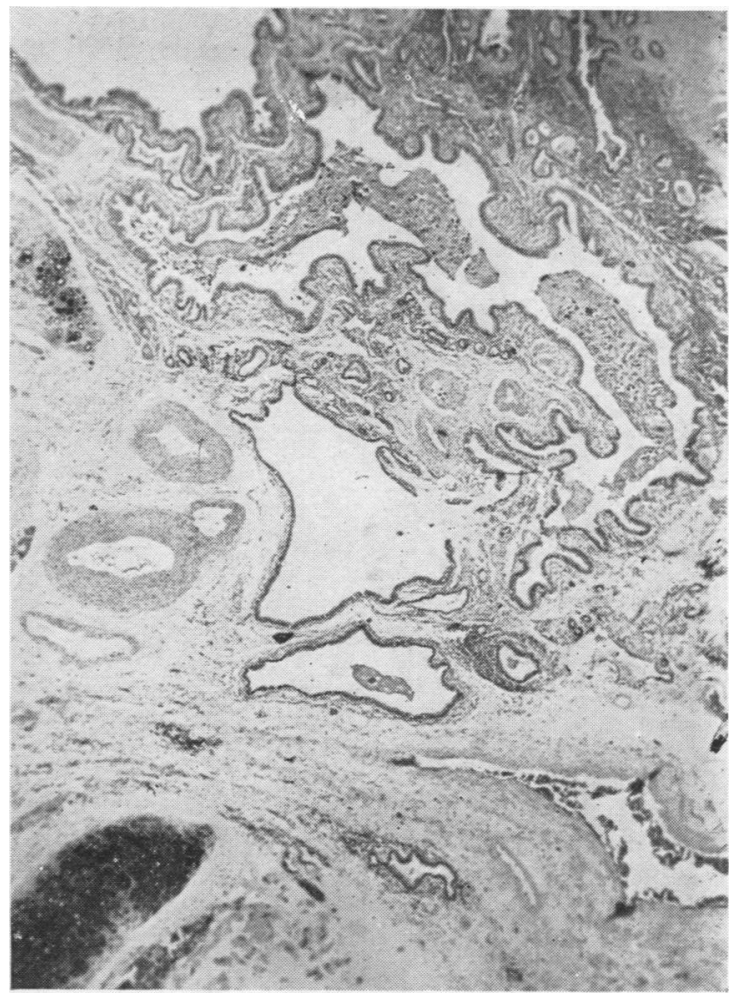

FIG. 4.-Transverse section of the loculated portion of the cyst stained by haematoxylin and eosin. There are many cystic spaces lined by pseudostratified ciliated columnar epithelium, producing an appearance suggestive of bronchi. Portions of cartilage can also be seen. 'Thick-walled blood vessels are present between the cystic spaces $(\times 25)$. 

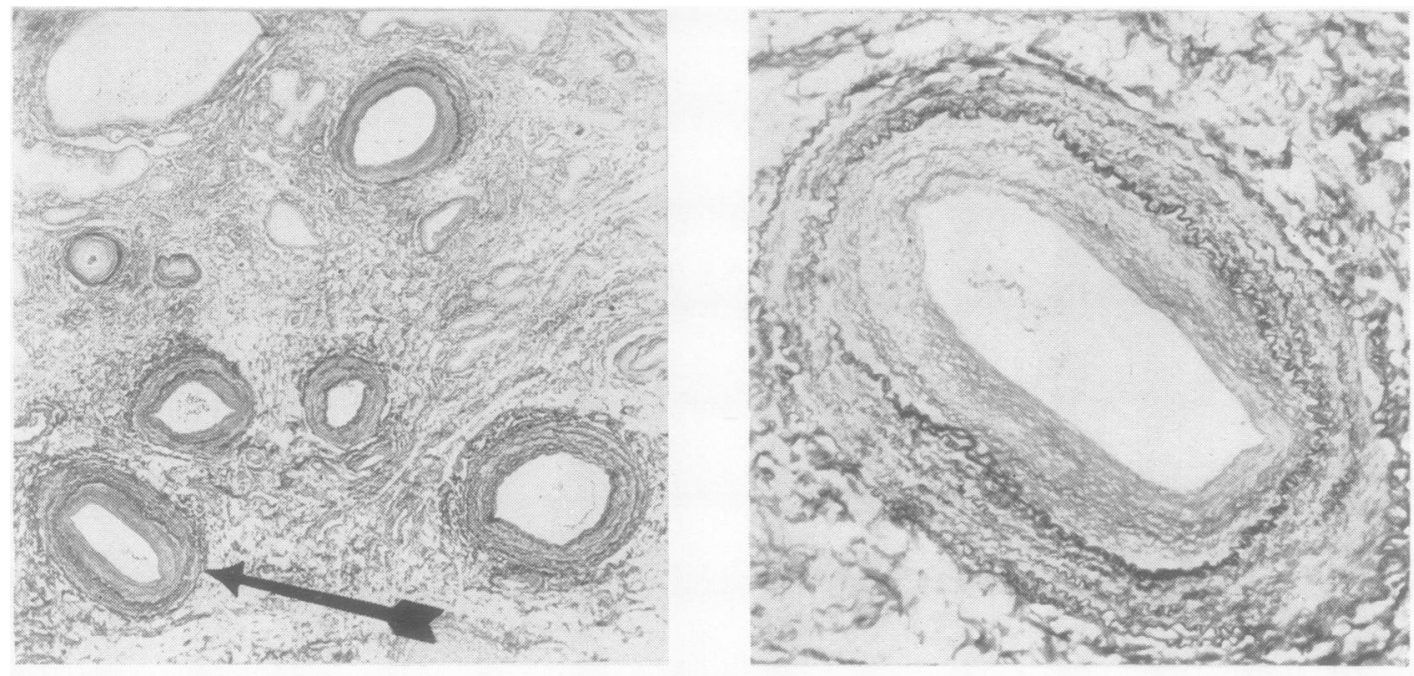

B

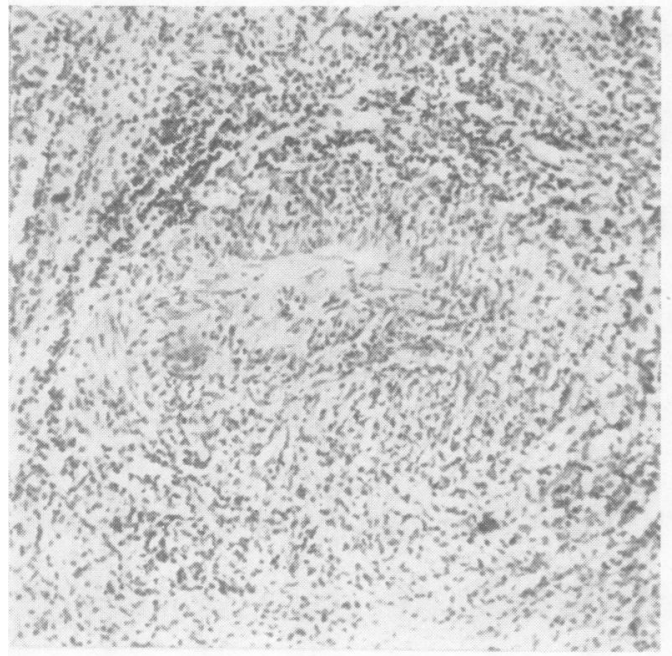

C

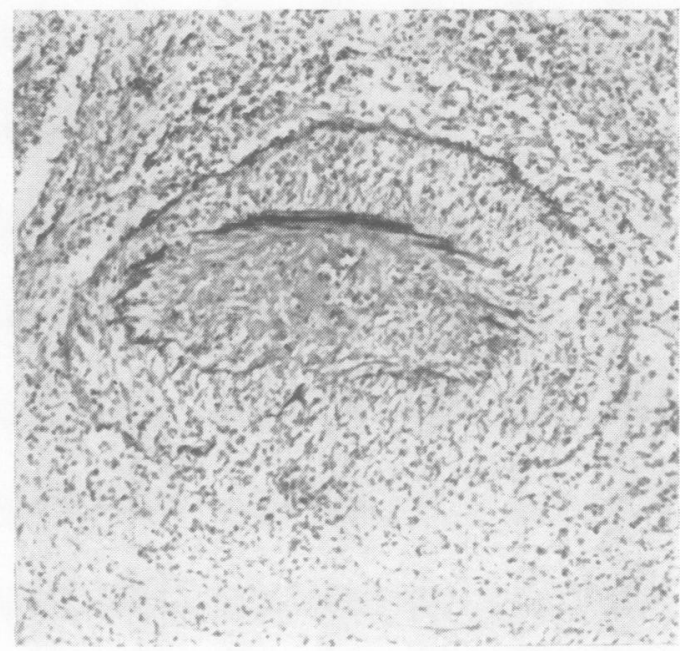

D

Fig. 5.-A. Section through the nodular part of the cyst wall showing systemic blood vessels, regarded as branches of the phrenic arteries. They show severe intimal fibrosis. The vessel indicated by a black arrow measures $660 \times 440 \mu$ and is shown at greater magnification in Fig. 5B $(\times 25)$. B. Transverse section of the blood vessel marked with a black arrow in Fig. 5A. The medial thickness is roughly $70 \mu$. There is severe intimal fibrosis with admixed elastic fibrils $(\times 100)$. Figs. 5A and B stained by Lawson's modification of Weigert-Sheridan method and van Gieson's stain. C. Transverse section of one of the systemic arteries in the loculated portion of the cyst, stained by haematoxylin and eosin, to demonstrate necrosis of the media and arteritis. All layers of the vessel are infiltrated with leucocytes. There is hyaline fibrous tissue in the lumen of the vessel $(\times 105)$. D. The same section shown in $5 \mathrm{C}$ stained to demonstrate elastic measures $500 \times 300 \mu$; its medial thickness is $80 \mu$ on the average, about $20 \%$ of the average diameter of the vessel. There is a rupture in the external elastic lamina $265 \mu$ in length and over the corresponding arc of the vessel the internal elastic membrane is thin and fragmented; portions of elastic fibrils can be seen in the necrotic media in this region $(\times 105)$. 


\section{Discussion}

The NAture of THE CyST.-The cyst appears to be an accessory lung for the following reasons: it is formed of anomalous, non-functioning pulmonary tissues, namely cystic bronchi and attenuated pulmonary alveoli, and hence has the characteristics of one of the forms of sequestration of lung. On the basis of the criteria held by Abbey Smith (1956) to differentiate between intralobular and "extralobular" sequestration the cyst represents the latter variety, more correctly termed an accessory lung. First it was not in the intralobular position in the posterior basic segment of the lower lobe of a lung, invariably taken by the intralobular type of sequestration, but was embedded in the diaphragm; accessory lungs have been reported in proximity to this muscle. Secondly, in the present patient there was an associated hiatus hernia, which commonly occurs with extralobular sequestration (Valle and White, 1947) but only exceptionally with the intralobular variety (case from Massachusetts General Hospital, 1943, mentioned by Abbey Smith, 1956). Furthermore there was no prominent single artery arising from the aorta to supply the cyst such as frequently occurs in intralobular sequestration but has been reported only once in accessory lung (Simpson, 1908). The cyst appeared to be vas-

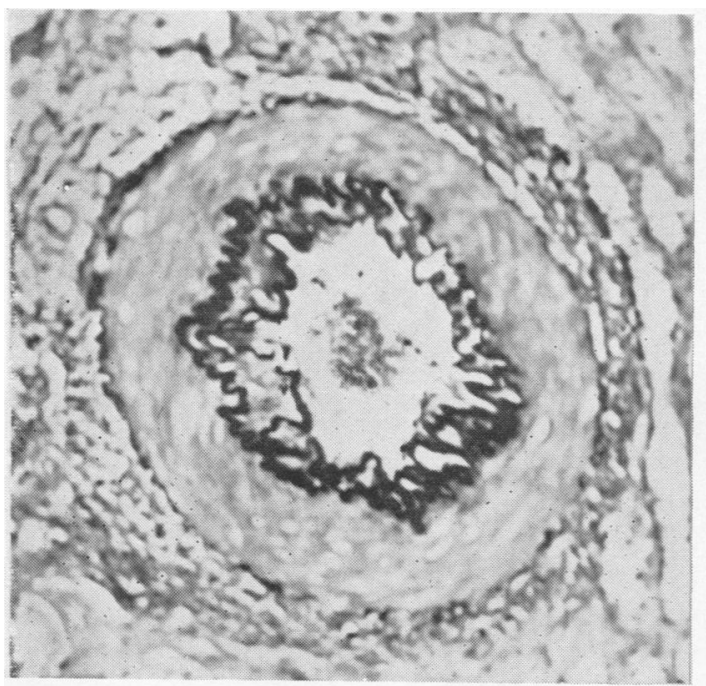

A

FIG. 6.-Left: Transverse section of a renal artery, $380 \times 320 \mu$, in systemic hypertension, illustrating the configuration of elastic tissue in systemic arteries of this diameter. There is a thick internal elastic membrane with splitting of elastic fibrils into the intima (elastosis); the external elastic membrane is also clearly visible but less prominent. There are no concentric elastic laminae in the media ( $\times 125$, Verhoeff's and van Gieson's stains). Right: Transverse section of the cyst artery, $800 \times 680 \mu$, shown in the bottom right-hand corner of Fig. 5A, illustrating the configuration of elastic tissue normally found in pulmonary vessels of this diameter. The internal and external elastic membranes are not more prominent than the concentric elastic laminae scattered through the media $(\times 85)$. Lawsen's modification of the Weigert-Sheridan method for elastic and van Gieson's stain.

cularized by small branches of the phrenic arteries, the nearest available blood supply, while the venous drainage was into the phrenic veins, as there was no possible communication with the pulmonary circulation. Intralobular sequestrations, on the other hand, have venous drainage into the pulmonary veins, the only exception to this being the case of Kergin (1952) which had a vein draining into the azygos system. There was admittedly no connexion between the cyst under discussion and the foregut but this is by no means invariable in accessory lung.

NATURe OF THE ARTeries IN THE CYST.-The arteries in the cyst are characterized by a thick media equivalent to 10 to $20 \%$ of the external diameter of the vessel which is composed of loosely packed smooth muscle and elastic fibrils arranged evenly in a concentric fashion throughout the media between the internal and external elastic membranes. They are not bronchial according to the criteria of Verloop (1948). Furthermore they are not characteristic of systemic vessels of a comparable size which have a media composed of more densely packed smooth muscle and a different arrangement of elastic tissue characterized by a frequently thin and ill-formed external elastic lamina and a thick internal lamina which may

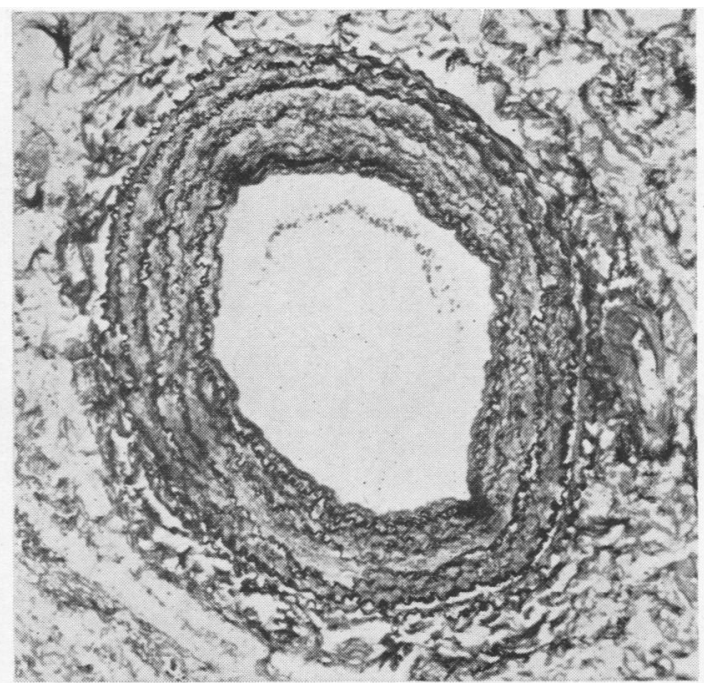

B 
show pathological splitting into the intima to produce the appearance of elastosis commonly seen in the renal vessels (Fig. 6). The appearances are more like those of systemic elastic arteries of an early order of branching from the main arterial trunks or of large muscular pulmonary arteries showing the features of hypertensive pulmonary vascular disease (Heath and Whitaker, 1956). The accessory lung was entirely intradiaphragmatic and had no connexion with the normal lungs and the pulmonary and bronchial circulations; it was found at operation to be supplied by small branches of the phrenic arteries. In undergoing this transition from supplying a small area of diaphragm to vascularize abnormal pulmonary tissue these systemic vessels appear to have acquired certain features, such as the multiple elastic laminae, which are abnormal for this size of vessel elsewhere in the systemic circulation but normal in the lung. Similarly, as described by Pryce (1946) and Abbey Smith (1956), intralobular sequestrations are also supplied by elastic systemic arteries.

Relation of Changes in Pulmonary Structures Forming THE CYST TO ITS TOTALLY SYSTEMIC BLOOD SUPPLY.-Abbey Smith (1956) has suggested that in intralobular sequestrations of lung there may be an excessive blood flow to the bronchi at the expense of the distal parts of the bronchopulmonary tree, which is deprived of a blood supply on account of the absent pulmonary vasculature. He considers that this may produce abnormal development and cystic expansion of the bronchi, as in the present case, together with underdevelopment of the pulmonary alveoli to the extent that the interbronchial tissue consists of nothing more than fibrous tissue containing foci of inflammatory cells. This thesis appears to be supported by the macroscopic and histological resemblance between sequestrations of lung and cystic bronchiectasis, for although bronchiectatic lung is connected with a normal pulmonary vasculature it has also an excessive systemic blood supply in the form of a bronchial collateral circulation. It could be argued that such bronchial dilatation and interstitial fibrosis are due to blood at high pressure and oxygen concentration supplying the conduction components of the lung at the expense of the parenchyma. However, since Baar and d'Abreu (1949) have reported similar microscopical findings in an example of accessory lung which was indisputably supplied by histologically normal pulmonary arteries the concept of a direct relation between a disproportionately large systemic blood supply and cystic dilatation of bronchi and alveolar underdevelopment, must be accepted with reserve. Identical histological changes in accessory lungs supplied solely by pulmonary or systemic arteries make it appear likely that such changes are primarily congenital anomalies rather than secondary to haemodynamic effects. Thus the report of Cole, Alley, and Jones (1951) of the association of aberrant systemic arteries to the lung originating from the lower thoracic or upper abdominal aorta, with accessory pulmonary lobes, cystic disease of the lower lobe and diaphragmatic defects adds further support to the idea that cystic changes in the lung in common with diaphragmatic hernia may arise as coincidental congenital findings with anomalous systemic vessels rather than as a direct haemodynamic result of totally systemic supply.

The Changes in the Systemic Arteries.-The occurrence of medial hypertrophy and intimal proliferation of fibrous tissue in systemic arteries supplying pulmonary tissue has been reported before by Cudkowicz and Armstrong (1951) who described these changes in the bronchial arteries in cases of emphysema. They found fibrosis of the interlobular septa, visceral pleura, and the smaller bronchi, all supplied by the histologically abnormal bronchial arteries, and "epithelial attenuation" in the peripheral alveoli, and concluded that occlusion of bronchial arteries leads to ischaemic changes in the areas supplied by them. In the present case it is possible, as already stated, that similar changes in the systemic arteries supplying the cyst led to similar fibrosis, but one objection to this, namely the occurrence of similar fibrotic changes in the stroma of accessory lungs supplied by normal pulmonary arteries, has already been brought forward.

The abnormal cystic vessels have a structure normally found in larger systemic arteries of an earlier order of branching from one of the major elastic trunks. This abnormal structure appears to have rendered the cyst vessels less able to cope with systemic blood pressure, and they have reacted to it in the same way as the pulmonary vasculature reacts to hypertension in the lesser circulation. The similarity between the vascular changes in the cyst as exemplified by intimal fibrosis and necrotizing arteritis and those of hypertensive pulmonary vascular disease is notable. Although there have been many reports of medial hypertrophy with intimal sclerosis and necrotizing arteritis in the pulmonary arteries in many forms of heart disease associated with pulmonary hypertension, there do not appear to be previous descriptions of similar changes in abnormal systemic vessels supplying aberrant pulmonary tissue. 


\section{SUMMARY}

A description is given of the pathology of a diaphragmatic cyst and changes, including arteritis, that occurred in the blood vessels supplying it. The nature of the cyst is discussed and reasons given for regarding it as an accessory lung (" extralobular sequestration "). The blood vessels supplying it were abnormal branches of phrenic arteries which had characteristics of larger radicles of major elastic systemic arteries of an earlier order of branching than would be appropriate for the site of the vessels in the posterior part of the diaphragm. The relation of changes in the pulmonary structures forming the cyst to its totally systemic blood supply is discussed. The vascular changes are discussed and considered to be analogous to those of hypertensive pulmonary vascular disease in that they illustrate that arteries supplying pulmonary tissue, be they pulmonary or abnormal systemic vessels, hypertrophy and undergo necrosis in reaction to a systemic blood pressure that they are structurally ill adapted to cope with.

\section{REFERENCES}

Baar, H. S., and d'Abreu, A. L. (1949). Brit. J. Surg., 37. 220. Cole, F. H., Alley, F. H., and Jones, R. S. (1951). Surg. Gyner. Obstet., $93,589$.

Cudkowicz, L., and Armstrong, J. B. (1951). Thorax, 6, 343.

Heath, D., and Whitaker, W. (1956). Circulation, 14, 323.

Kergin, F. G. (1952). J. thorac. Surg., 23, 55.

Pryce, D. M. (1946). J. Path. Bact., 58, 457.

Simpson, G. C. E. (1908). J. Anat. (Lond.), 42, 221.

Smith, R. Abbey (1956). Thorax, 11, 10.

Valle, A. R., and White, M. L. (1947). Dis. Chest, 13, 63.

Verloop, M. C. (1948). Acta anat. (Basel), 5, 171. 